The Chittagong Univ. J. Sci. $40: 21-46,2018$

\title{
Greenhouse Gas Emissions from Municipal Solid Waste Management of the Chittagong City Corporation
}

\author{
Md. Danesh Miah* and M Abubokor Siddik \\ Institute of Forestry and Environmental Sciences, University of Chittagong, \\ Chittagong 4331, Bangladesh \\ *Corresponding author:dansmiah@gmail.com;danesh@cu.ac.bd
}

\begin{abstract}
Municipal solid waste (MSW) management has an impact on climate generating greenhouse gases (GHG). To quantify GHG emission from MSW management activities (transportation, composting, recycling and landfill), this study was conducted in the Chittagong City Corporation (CCC). Data were collected about detail MSW management activities of the CCC through conducting a structured questionnaire survey on related personnel with MSW management. To collect data about recycling, another questionnaire survey was conducted on all junkshops near to both dumping sites (Anandabazar and Arefin Nogor) of the CCC. The study found that, composting and recycling of MSW have a positive contribution in reducing GHG emission. Transportation and land-filling of MSW have a contribution in GHG emission. The amount of GHG emission from the existing MSW management system of the $\mathrm{CCC}$ is $31,904.68$ tons of $\mathrm{CO}_{2}$-eq per month. The life cycle assessment (LCA) study on composting shows that, the reduction of GHG emission is 3.66 tons of $\mathrm{CO}_{2}$-eq per ton of produced compost. The study finding is expected to contribute to the field of climate change mitigation in Bangladesh.
\end{abstract}

Keywords: Composting; Recycling; Transportation; Land-filling; Life Cycle Assessment 
22 Md. Danesh Miah and M Abubokor Siddik

গ্রীণহাউজ গ্যাস উদ্শীরণের মাধ্যনে মহানগর কঠিন বর্জ্য ব্যবস্থাপনা জলবায়ুর উপর প্রভাব রাখে। মহানগর কঠিন বর্জ্য ব্যবস্থাপনার (পরিবহন, কস্পোস্টকরন, পুনরাবর্তন এবং ল্যান্ডফিল) মাধ্যন্মে গ্রীণহাউজ গ্যাস উদ্শীরণ নির্ধারণ করার জন্য চট্টগ্রাম মহানগরে এই গবেষণাটি সম্পন্ন করা হয়। মহানগর কঠিন বর্জ্য ব্যবস্থাপনা’র বিস্তারিত জানার জন্য সম্পর্কযুক্ত ব্যক্তিদের থেকে একটি গঠনগত প্রশ্নমালা জরিপের মাধ্যমে গবেষণাটি সম্পন্ন করা হয়। কঠিন বর্জ্যের পুনরাবর্তন সম্পর্কে ডাটা সংগ্রহ করার জন্য কঠিন বর্জ্যের ডাম্পিং সাইটের (আনন্দবাজার এবং আরেফিন নগর) সকল জাংশপগুলোকে নিয়ে আরেকটি প্রশ্নমালা জরিপ সম্পন্ন করা হয়। গবেষণার ফলাফলের মতে, কস্পোস্টিং এবং কঠিন বর্জ্যের পূনরাবর্তন গ্রীণহাউজ গ্যাস হ্রাস করার ক্ষেত্রে ধনাত্নক প্রভাব রাখে। কিন্তু কঠিন বর্জ্যের পরিবহন এবং ল্যাণ্ডফিলিং গ্রীণহাউজ গ্যাস নির্গমনে সহায়ক ভূমিকা রাখে। মহানগর কঠিন বর্জ্য ব্যবস্থাপনার বর্তমান অবস্থা প্রতিমাসে ৩১,৯০৪.৬৮- টন কার্বন ডাই অক্সাইড ইকুইভ্যালেন্ট নির্গমন করে। আবার প্রতি টন কম্পোস্ট তৈরি করার ক্ষেত্রে ৩.৬৬ টন কার্বন ডাই অক্সাইড ইকুইভ্যালেন্ট নির্গমণ হ্রাস করে। বাংলাদেশের মাধ্যনে জলবায়ু পরিবর্তন নিবৃক্তির ক্ষেত্রে এই গবেষণাটি অবদান রাখবে।

\section{Introduction}

Municipal solid waste management (MSWM) is one of the most important issues due to its annual generation rate (2-3\%) for developing countries [1]. It is also subject of global concern as a high contributor to global greenhouse gas (GHGs) emission [2-6], which is a significant environmental issue worldwide [7]. Because, GHGs contribute to global warming causing climate change.

Solid waste management activities, such as waste transportation, composting, incineration, open burning, mix-waste land-filling, anaerobic 
digestion, mechanical biological treatment emits GHGs [3, 4, 6]. GHG emission from waste transportation is 39.14 thousand tons of $\mathrm{CO}_{2}$-eq in Hangzhou, China [8]. Composting not only emits GHG to atmosphere, but also reduces GHG emission through using compost in agricultural sector by reducing the use of chemical fertilizer. GHG emission is 82.21 thousand tons of $\mathrm{CO}_{2}$-eq from food waste composting in Hangzhou, China [8]. Waste composting reduces 203,409 tons of $\mathrm{CO}_{2}$-eq GHG emission in Edmonton, Canada per year [9]. The contribution of waste sector is $13 \%$ of total GHG emissions in Israel [10]. Approximately, 30 to $40 \%$ of the total anthropogenic methane $\left(\mathrm{CH}_{4}\right)$ emits from waste management in Finland [11].

The largest contributor to global warming from MSWM sector is landfill due to emission of landfill gas [8, 12]. Landfill gas (LFG) mainly consists of methane $\left(\mathrm{CH}_{4}\right)$ and carbon dioxide $\left(\mathrm{CO}_{2}\right)$, with small fraction of hydrogen sulfide $\left(\mathrm{H}_{2} \mathrm{~S}\right)$, nitrogen $\left(\mathrm{N}_{2}\right)$ and volatile organic compound (VOCs) $[4,7,13]$. Due to high emission of $\mathrm{CH}_{4}$ among these GHGs, landfill has been ranked as the third largest anthropogenic $\mathrm{CH}_{4}$ emission sources [14, 15]. Worldwide GHG emission from landfills ranged from 9 to $70 \mathrm{Tg}$ annually [14] and 747.4 tons $\mathrm{CO}_{2}$-eq in 2005 [16]. Yee et al. [9] estimated that, GHG emission from Clover Bar Landfill, Edmonton, Canada is 32,848 tons of $\mathrm{CO}_{2}$-eq in 2011 .

In annual global anthropogenic $\mathrm{CH}_{4}$ emission, the contribution of landfill is about 3-19\% [15, 17]. Methane emission is 8.46 tons in 1997 [18]; 171 tons in 1999 [19]; 115400 tons in 2004 and 89220 tons in 2006 [20, 21] from different landfill of China, Taiwan, India and Thailand, respectively. Talyan et al. [22] projected that, the methane emission will be 254000 tons per year by the year 2025 
and 339000 tons per year by the year 2020 [20] from landfill sites of Delhi and Thailand, respectively. Carbon dioxide emission from landfills was 828 tons in 1999 in Taiwan [19].

To assess environmental impacts of current and possible improvements of MSWM system, recent researches focus on LCA [12, 23-29]. Some studies assess the comparison of different environmental performance with the introduction of recycling scenerios [30-33]. Some studies also exist based on LCA dealing with the analysis of GHG emissions, both from the management of mixed solid waste perspective [29, 34-36] and individual solid waste management method such as incineration, composting, anaerobic digestion, land-filling [27, 37-39].

While reviewing the literatures, several studies were found on - solid waste generation and management facilities [40-44], on solid waste recycling [40, 42, 4547] and on development of model for sustainable solid waste management in Bangladesh [48]. But there have no study on quantification of GHG emission from solid waste management facilities in Bangladesh. So, this study was undertaken to quantify the amount of GHG emission from MSWM facilities of Chittagong City Corporation with the specific objectives of assessing the status of existing MSW disposal system; quantifying GHG emission from the existing different disposal methods of MSW; and assessing the LCA of composting. The study will be helpful to select proper treatment facilities for MSWM to reduce GHG emission and to the climate change mitigation practitioners in Bangladesh.

\section{Materials and Method}

The study was conducted in the Chittagong City Corporation (CCC) area during January 2014 through August 2014. 


\section{Background information}

\section{Description of the study area}

Chittagong is the second largest city and also known as the commercial capital of Bangladesh. It is bounded on the south by the Anowara and Patiya Upazilas, on the east by the Patiya and Boalkhali Upazilas, on the north by the Hathazari and Sitakunda Upazilas and the west by the Bay of Bengal (Figure 1). The land area of Chittagong city is 157 square kilometer (61 square miles). It is located between $22^{0} 14^{\prime}$ and $22^{0} 30^{\prime}$ north latitudes and between $91^{\circ} 45^{\prime}$ and $91^{\circ} 53^{\prime}$ east longitudes [49]. In Chittagong city, mean annual rainfall is $3058 \mathrm{~mm}$ with mean annual temperature $29.53^{\circ} \mathrm{C}$ having a mean annual relative humidity $78 \%$ [49]. According to the Population Census 2011, total population of Chittagong city is $25,92,439$ having male $13,67,852$ and female $12,24,587$ with a population density 16513 per $\mathrm{km}^{2}$ in the total number of households 5,58,097 [50]. The literacy rate of the people of Chittagong city is $69 \%$. Average household size is 4.6 and annual growth rate of population is 2.67 [50]. The contribution of Chittagong city on national economy is much higher than the other cities because of Chittagong port, diversified economic activities, natural beauties, industrial activities and its suitable geographical location factor in the regional map. In Chittagong, the major economic settlements are (a) Chittagong port, (b) lots of garments industries, (c) huge number of medium and heavy industries (industrial belt at Fouzdarhat, Baizid Bostami, Kalurghat industrial and Potenga industrial area), and (d) natural beauties such as Potenga sea beach, Foy's lake, Karnaphuly river bank, Batali hill. Natural beauties are playing vital role in the development of tourism industry in Chittagong. It was upgraded as a municipal city in 1863, and 
was upgraded to a municipal corporation in 1982 and finally to a city corporation in 1989. There are 41 wards (administrative areas) in Chittagong city. According to the Chittagong City Corporation (CCC) record book, at present total population of Chittagong city is 6.5 million (Pers. com $^{l}$ ). Having a huge population and dense commercial activities, the CCC is incorporating a huge solid waste dumping and concerning its management too.

Existing municipal solid waste management system of the Chittagong City Corporation

The existing MSW management system includes solid waste collection, composting, recycling of inorganic waste and land-filling. Among these, recycling of inorganic waste is completely done by informal private sector. Waste pickers (locally called "Tokai") are engaged in collection of recyclable waste materials from collected waste of the CCC in two dumping (Anandobazar and Arefin Nogor) sites. All collected recyclables are sold to locally available shop known as "Vangari" or "Scrap dokan" (all of these shops near to the dumping site). Other activities, such as collection, composting and land-filling are done by the CCC.

\footnotetext{
${ }^{1}$ Data was collected from the office of Chittagong City Corporation, Chittagong.
} 


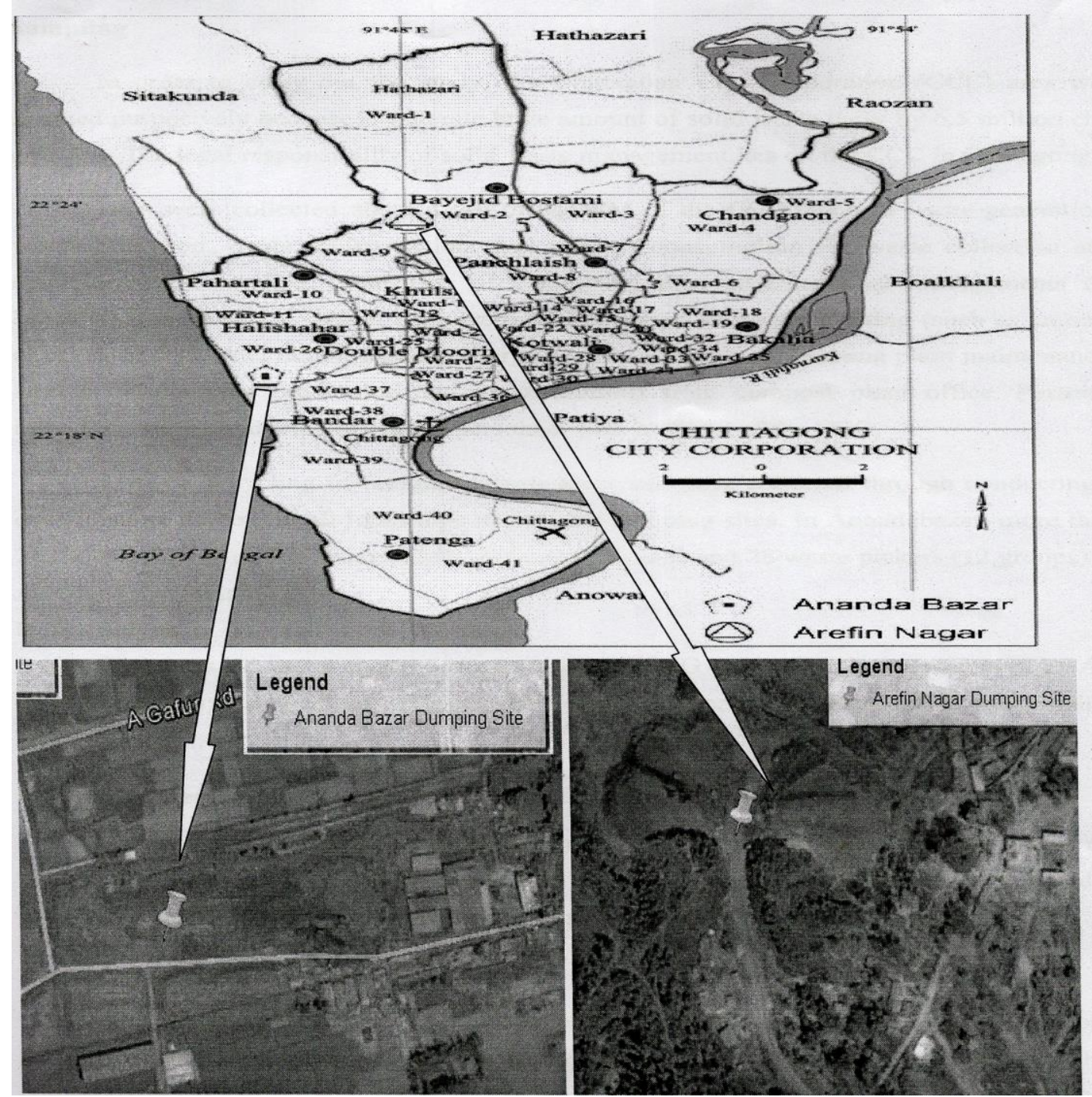

Figure 1: Map of the study area showing Chittagong City Corporation. 


\section{Sampling}

In order to carry out the study, the Chittagong City Corporation (CCC) area was selected purposively because it generate huge amount of solid waste daily by 6.5 million city dwellers. The legal responsibility of solid waste management lies on the CCC in Chittagong.

Data were collected about detail of MSWM of the CCC (such as waste generation, waste collection, waste transportation, fuel (diesel) consumption for waste collection and operational maintenance, population of Chittagong city, organizational arrangements for MSWM) from the CCC office. Data were also collected about composting (such as amount of organic waste used in composting, energy consumption for composting plant maintenance, amount of compost production and its utilization) from compost plant office. Personal interviews were conducted of related personnel with MSWM of the CCC.

Data on recycling of inorganic waste materials were collected through conducting a questionnaire survey on all junkshops near to the dumping sites. In Anandabazar, more than 200 waste pickers collects recyclables under 8 junkshops and 28 waste pickers (12 groups) in Arefin nogor dumping sites.

\section{Data analysis}

Data were processed and analyzed for assessment of GHG emission and assessment of LCA of composting by using Microsoft Excel 2007, and "GHG calculator for Solid Waste-Version II" [51]. 


\section{Life cycle assessment (LCA)}

Environmental impacts of compost production from degradable organic waste are done in Chittagong. LCA has been used as the evaluation tool. Each phase of LCA adopted in this study is explained in the following sections.

\section{Goal and scope definition}

The goal of this study is the assessment of environmental impacts of compost production of the $\mathrm{CCC}$ from a life cycle perspective and assessment of GHG emissions/savings of per unit produced compost. Environmental impacts of composting were evaluated by considering its air emissions, energy (fuel and electricity) consumption and produced compost. The components of composting system were considered in this scenario: collections, transportation, separation, degradation of waste and compost plant maintenance. The system boundary is shown in Figure 2.

Inventory analysis

Inventory analysis involves with actual data collection, validation and calculation procedures. In this phase, all data about composting (amount of organic waste used for composting, fuel consumption for collection, and electricity consumption for operational maintenance) were collected from the office of the CCC. GHG emission from composting system was calculated by using GHG emission factors for MSW composting from IPCC waste model [3]. 


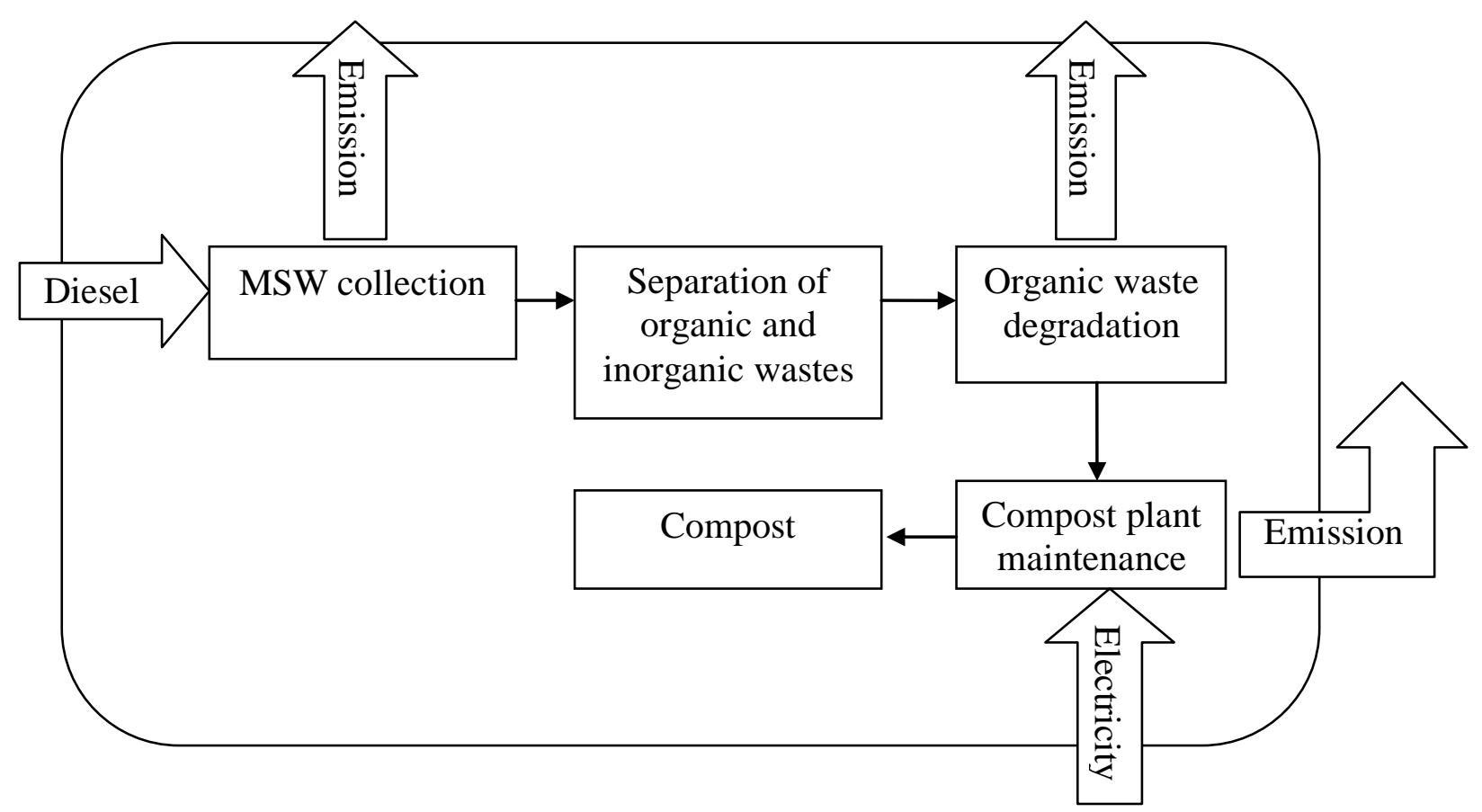

Figure 2: System boundary of LCA study of compost in the Chittagong City Corporation.

\section{Impact assessment}

Potential environmental impacts are calculated based on the data from the inventory analysis. Many impacts assessment methodologies are developed in compliance with the ISO 14040 framework. The "GHG calculator for Solid WasteVersion II" method was adopted in this study [51]. In this study, impacts category considered is only GHG emissions in the evaluation.

\section{Interpretation}

Finally, useful results from inventory analysis and impact assessment are discussed in accordance with the goal and scope of the study. 


\section{Results and Discussion}

Status of existing municipal solid waste disposal system of the Chittagong City Corporation (CCC)

The municipal solid waste (MSW) management system of the CCC was consisted of three steps. These are collection, composting and land-filling.

\section{Collection and disposal of municipal solid waste}

Total MSW generation is about 1938 tons per day with per capita generation rate $0.30 \mathrm{~kg}$ in the CCC. The CCC collects $1357 \pm 151.2$ tons waste daily (approximately $70 \%$ of the generated waste) through the existing waste collection facilities of the CCC. Among the collected wastes, $0.44 \%$ (6 tons) is disposed to composting, $0.45 \%$ (6.1 tons) to recycling of inorganic waste, and rest $99 \%$ (1344.9 tons) are landfilled (Figure 3).

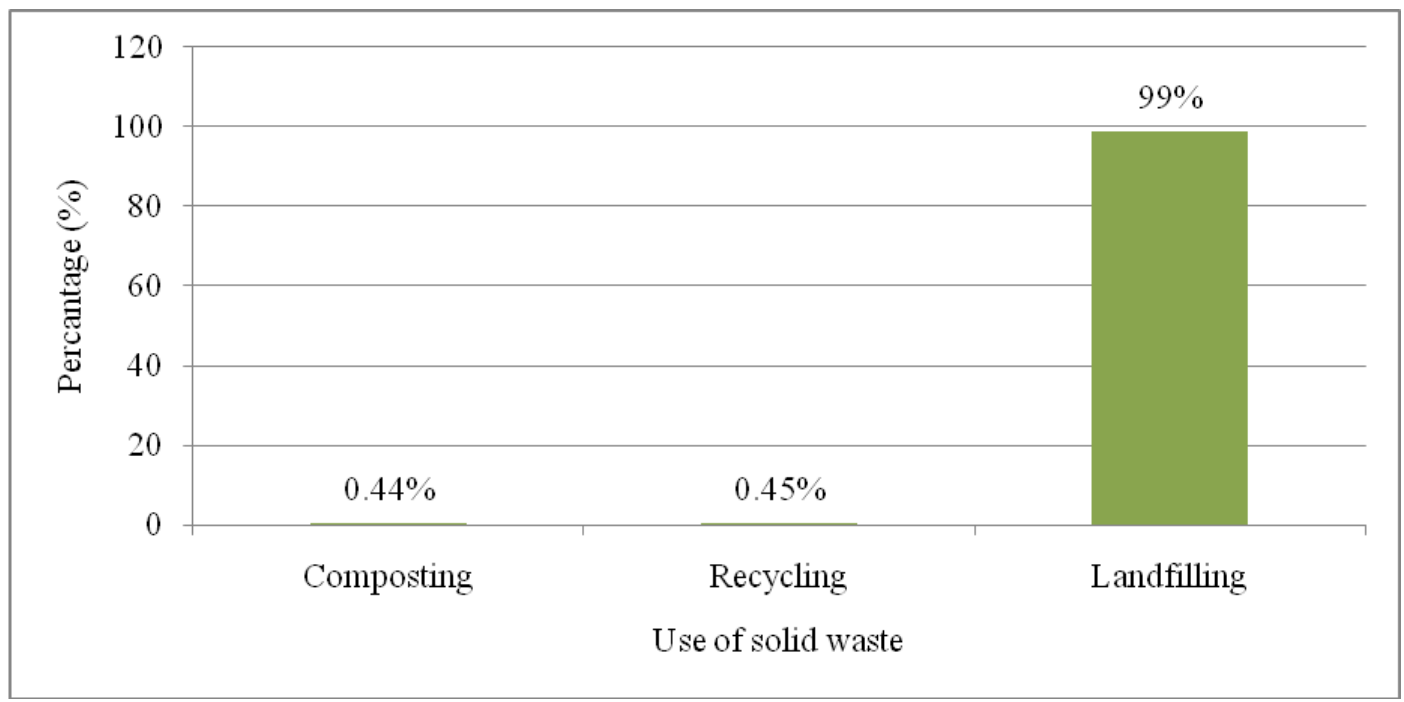

Figure 3: Use of municipal solid waste in the Chittagong City Corporation. 
Enayetullah et al. [42] reported that, waste generation was 4634 tons, 1548 tons, 172 tons, 321 tons, 134 tons, and 142 tons, respectively, in Dhaka, Chittagong, Rajshahi, Khulna, Barisal and Sylhet. BMDF [40] also reported that, waste generation rate (WGR) was $0.34 \mathrm{~kg} / \mathrm{capita} /$ day in Chittagong city. Recent data on the per capita WGR in some Asian countries are close to the WGR of Chittagong. For example, per capita WGR are 0.46, 0.65, 0.70, 0.337 and 0.565 kg/day in India, Malaysia, Indonesia, Sri Lanka and Nepal, respectively [52]. However, the WGR of Chittagong is very different to some other countries, such as the USA, the EU, Turkey and Mexico, where per capita waste generation rate is 2.08, 1.51, 0.97 and $0.92 \mathrm{~kg} /$ day, respectively [53]. Enayetullah et al. [42] also reported that, waste collection efficiency was 37\%, 70\%, 56\%, 47\%, 44\%, $76 \%$ in Dhaka, Chittagong, Rajshahi, Khulna, Barisal and Sylhet, respectively. In some state of India, waste collection efficiency have been observed - 82\%, 80\%, 74\%, $72 \%$ and $71 \%$ for Kerala, Karnataka, West Bengal, Maharashtra and Punjab, respectively [54].

\section{Recycling of municipal solid waste}

Recycling of municipal solid waste is another part of solid waste management of the CCC. But there is no formal organization in Chittagong for MSW recycling. Informally, waste pickers (locally called Tokai) are engaged in collection of recyclable waste materials from collected waste of the CCC from two dumping sites (viz. Anandobazar and Arefin Nogor). All collected recyclables are sold to locally available shop known as "Vangari or Scrap dokan" (most of these shops near to the dumping site). The findings of the conducted survey along with 
the current study to assess recycling details at two dumping sites of the CCC (viz. Anandobazar and Arefin Nogor) presented at the Table 1. It reveals that, the waste pickers collect a total about 6.102 tons of recyclable materials from the two dumping sites (4.758 ton from Anandobazar and 1.344 ton from Arefin Nogor), which is only $0.45 \%$ of total daily collected waste of the CCC.

Sujauddin et al. [47] found that, amount of recycling of paper, plastic, glass, steel, bone and coconut shell was $958 \mathrm{~kg}, 1084 \mathrm{~kg}, 1586 \mathrm{~kg}, 150 \mathrm{~kg}, 345 \mathrm{~kg}$ and $545 \mathrm{~kg}$, respectively in Chittagong city. Chowdhury et al. [41] found that, total amount of recyclables was $3302 \mathrm{~kg}$ in Chittagong city. Alamgir and Ahsan [45] found that, the amount of recyclables was $1,741,730$ tons in six major cities of Bangladesh in 2005. The amount of recovered materials such as plastic, paper, glass and cardboard was 1.5 tons per month in Galle, Sri Lanka [55]. By $40 \%$ and 53\% increasing recycling of MSW in Japan and South Korea, both countries reduced solid waste generation by $20 \%$ and $12 \%$, respectively [52]. Troschinetz and Mihelcic [53] found that, the resource recovery rate through recycling was 5$40 \%$ of total generated waste in developing countries, which consisting $0-70 \%$ recyclables and $17-80 \%$ organic materials. 
Table 1. Daily municipal solid waste recycling scenario by waste pickers at Anandobazar and Arefin Nogor dumping site in 2014 under the Chittagong City Corporation.

\begin{tabular}{lccc}
\hline \multirow{2}{*}{$\begin{array}{c}\text { Recyclable } \\
\text { materials }\end{array}$} & \multicolumn{2}{c}{ Average daily collection (ton) } & Total daily collection \\
\cline { 2 - 3 } & Anandobazar & Arefin Nogor & (ton) \\
\hline Paper & 1.575 & 0.284 & 1.859 \\
Plastic & 0.550 & 0.476 & 1.026 \\
Aluminum & 0.027 & 0.011 & 0.038 \\
Steel & 0.556 & 0.132 & 0.688 \\
Glass & 2.050 & 0.441 & 2.491 \\
\hline Total & 4.758 & 1.344 & 6.102 \\
\hline
\end{tabular}

\section{Greenhouse gas emission from municipal solid waste management}

GHG emission is occurred from different stages of existing MSWM system of the CCC. These stages are waste transportation, composting, recycling of recyclable materials and land-filling. The Table 2 represents amount of GHG emission per ton of waste from each stage of existing MSWM system of the CCC. Description of GHG emission from each stage of MSWM system is given sequentially below.

\section{Greenhouse gas emission from waste transportation}

The daily MSW collection of the CCC is about 1357 tons (40924 tons per month) through 294 trips (8868 trips per month), which consumes 2552 litters diesel daily (76971 liters per month). Combustion of diesel caused GHG emission 
to the atmosphere. Direct GHG emission from waste transportation of the CCC is about 0.005 tons of $\mathrm{CO}_{2}$-eq per ton of waste (Table 1). Total GHG emission from waste transportation per month is about 207.443 tons of $\mathrm{CO}_{2}$-eq (Table 2).

Waste transportation caused huge GHG emission to the atmosphere all over the world. Different studies found different amount in different countries. Chen and Lin [23] found that, GHG emission from waste transportation was $4.4679 \mathrm{~kg}$ carbon equivalent (CE) per ton of waste collection. Total GHG emission from waste transportation was 39.14 thousand tons of $\mathrm{CO}_{2}$-eq in Hangzhou, China [8]; 2,747.23 metric ton carbon equivalent (MTCE) in Taipei, Taiwan [23]; 1 million metric ton carbon equivalent (MMTCE) in the United States [56].

Table 2. Greenhouse gas (GHG) emission per ton of waste from existing municipal solid waste management system of the Chittagong City Corporation.

\begin{tabular}{|c|c|c|c|c|}
\hline Process & $\begin{array}{l}\text { Direct GHG } \\
\text { emission }\end{array}$ & $\begin{array}{l}\text { Indirect GHG } \\
\text { savings }\end{array}$ & $\begin{array}{l}\text { Net GHG } \\
\text { emission }\end{array}$ & Unit \\
\hline Transportation & 0.005 & 0.00 & 0.005 & ton of $\mathrm{CO}_{2}-\mathrm{eq} / \mathrm{ton}$ of waste \\
\hline Composting & 0.177 & 1.314 & -1.137 & ton of $\mathrm{CO}_{2}-\mathrm{eq} / \mathrm{ton}$ of waste \\
\hline Recycling & 1.106 & 2.170 & -1.064 & $\begin{array}{l}\text { ton of } \mathrm{CO}_{2} \text {-eq/ton of } \\
\text { recyclables }\end{array}$ \\
\hline Land-filling & 0.791 & 0.00 & 0.791 & $\begin{array}{l}\text { ton of } \mathrm{CO}_{2} \text {-eq/ton of mix } \\
\text { waste }\end{array}$ \\
\hline
\end{tabular}


Md. Danesh Miah and M Abubokor Siddik

\section{Greenhouse gas emission from composting}

Composting is an effective treatment method for MSW. Organic portion of collected MSW can be efficiently managed through composting. The CCC managed about 180 tons of organic waste $(0.44 \%$ of collected waste) monthly through composting. Through composting process of the CCC, direct GHG emission is about 0.177 tons of $\mathrm{CO}_{2}$-eq per ton of organic waste used for composting (Table 2). The produced compost is used in agriculture sector, as a result composting also avoided GHG emission by replacing fertilizer and organic waste land-filling. Indirect $\mathrm{GHG}$ saving is about 1.314 tons of $\mathrm{CO}_{2}$-eq per ton of organic waste used for composting (Table 2). A net GHG emission from composting is about -1.137 tons of $\mathrm{CO}_{2}$-eq per ton of organic waste used for composting in life cycle perspective (minus GHG means potential savings) (Table 2). The total GHG emission from composting per month is about -204.716 ton of $\mathrm{CO}_{2}$-eq (Table 3).

In comparison to different cities of India, the composting efficiency of the CCC is very poor. Several Indian cities such as Delhi, Bangalore, Ahmedabad, Hyderabad, Bhopal, Luknow and Gwalior treat $9 \%$ of MSW by composting [54]. Dong et al. [8] in China and Yee et al. [9] in Canada also found that composting can reduce GHG emission. The amount of GHG emission reduction through composting was 82.21 thousand tons of $\mathrm{CO}_{2}$-eq in China [8] and 203,409 tons of $\mathrm{CO}_{2}$-eq in Canada [9]. 
Table 3. Monthly greenhouse gas (GHG) emission from existing municipal solid management system of the Chittagong City Corporation.

\begin{tabular}{lll}
\hline Process & Net GHG emission & Unit \\
\hline Transportation & 207.443 & ton of $\mathrm{CO}_{2}$-eq \\
Composting & -204.715 & ton of $\mathrm{CO}_{2}$-eq \\
Recycling & -194.750 & ton of $\mathrm{CO}_{2}$-eq \\
Land-filling & $32,096.703$ & ton of $\mathrm{CO}_{2}$-eq \\
\hline Total & $31,904.681$ & ton of $\mathrm{CO}_{2}$-eq \\
\hline
\end{tabular}

\section{Greenhouse gas emission from recycling of municipal solid waste}

Through recycling of recyclable materials about 183 tons of collected MSW is managed in Chittagong monthly. It is also an efficient method for avoiding GHG emission through life cycle perspective. Direct GHG emission is about 1.106 tons of $\mathrm{CO}_{2}$-eq per ton of mixed recyclable (Table 3). Indirect GHG saving is about 2.170 ton of $\mathrm{CO}_{2}$-eq per ton of mixed recyclable (Table 4). A net GHG emission from composting is about -1.064 ton of $\mathrm{CO}_{2}$-eq per ton of mixed recyclable in life cycle perspective (Table 4). The total GHG emission from recycling of inorganic materials per month is about -194.750 ton of $\mathrm{CO}_{2}$-eq (Table 3).

Several studies found the similar result that, recycling reduced GHG emission through avoiding virgin materials. Weitz et al. [56] found that, GHG avoidance through recycling from avoiding virgin materials were 4 MMTCE in United States. Chen and Lin [23] also found that, GHG avoidance through recycling were 24014.57 MTCE in Taipei, Taiwan. 
Md. Danesh Miah and M Abubokor Siddik

\section{Greenhouse gas emission from land-filling}

Land-filling is the main waste disposal method of the CCC, because the CCC disposes $99 \%$ of its collecting waste through this method. Landfill is the largest contributor to global warming among all GHG emission sources of existing MSW management system of the CCC. Direct GHG emission from waste landfilling of the $\mathrm{CCC}$ is about 0.791 tons of $\mathrm{CO}_{2}$-eq per ton of mixed landfill waste (Table 2). The total GHG emission from land-filling per month is about 32,096.703 tons of $\mathrm{CO}_{2}$-eq (Table 3).

In several cities of India, the municipality disposed their $90 \%$ of collected waste through landfill, which raise serious environmental degradation [54]. Several studies found that land-filling of MSW is highly responsible for GHG emission to the atmosphere. GHG emission from landfill was 32,848 tons of $\mathrm{CO}_{2}$-eq in Canada [9] and 234 teragrams (Tg) of $\mathrm{CO}_{2}$-eq in United States [56], 344.16 MTCE in Taipei, Taiwan [23]. Methane emission from landfill was 8.46 tons in China [18], 400660 tons in India [15], 115400 tons [20] and 89220 tons [21] in Thailand.

\section{LCA of composting}

Based on the goal and scope of the study, the results of the inventory analysis as well as the impact assessment are presented in this section.

\section{Impact assessment}

Composting is the only one treatment method used in management of solid waste of the CCC. This composting and garbage treatment plant (CGTP) was established in 2005 near Anandobazar dumping site. Only 0.44\% (6 tons) of total 
daily collected waste of the CCC mainly food and garden waste, is transferred to this compost plant. Composting itself and related activities - waste transportation, organic waste separation, transfer of waste to compost plant, compost plant maintenance - are included in the system boundaries. Diesel is used for start up of the composting (waste transportation). Electricity is used for compost plant maintenance from national grid. In this CGTP, monthly 180 tons of organic waste are treated, which generates 35 tons compost per month. The $100 \%$ of produced compost is used for agricultural purposes.

The findings of inventory analysis show that, among system boundary three stages such as collection of waste, waste degradation and electricity utilization for compost plant management responsible for GHG emission. The avoidance of landfilling of organic waste is caused reduction of GHG emission due to composting.

Potential environmental impacts of composting are shown in the Table 4. Waste required for compost plant consumed 338 litter of diesel for transportation monthly. Due to the combustion of diesel, the amount of GHG emission is 0.913 ton of $\mathrm{CO}_{2}$-eq monthly (Table 4). During composting, degradation of waste (using for composting) causes emission of huge amount of $\mathrm{CH}_{4}$ which is equivalent 31.860 tons of $\mathrm{CO}_{2}$-eq per month in compost plant. Compost plant maintenance consumes $750 \mathrm{kWh}$ electricity per month, which emits 0.443 tons of $\mathrm{CO}_{2}$-eq $\mathrm{GHG}$ during electricity generation. As a result of composting, 180 tons of organic waste is avoided from land-filling which caused avoidance of 161.280 tons of $\mathrm{CO}_{2}$-eq GHG. Finally composting reduces 128.065 tons of $\mathrm{CO}_{2}$-eq $\mathrm{GHG}$. Through impact assessment of composting, it is found that per ton produced compost reduced 3.66 tons of $\mathrm{CO}_{2}$-eq $\mathrm{GHG}$. 
Md. Danesh Miah and M Abubokor Siddik

Table 4. Emission of greenhouse gas (GHG) from different stages of composting in the Chittagong City Corporation.

\begin{tabular}{c|lrr}
\hline \multicolumn{1}{c|}{ Scenario } & \multicolumn{1}{c}{ Activity } & $\begin{array}{r}\text { Net GHG } \\
\text { emission }\end{array}$ & \multicolumn{1}{c}{ Unit } \\
\hline \multirow{2}{*}{ Composting } & Transportation & 0.913 & ton of $\mathrm{CO}_{2}$-eq \\
& Waste degradation & 31.860 & ton of $\mathrm{CO}_{2}$-eq \\
& $\begin{array}{l}\text { Compost plant } \\
\text { maintenance }\end{array}$ & 0.443 & ton of $\mathrm{CO}_{2}$-eq \\
\hline Non-composting & Land-filling of organic & -161.280 & ton of $\mathrm{CO}_{2}$-eq \\
& waste & -128.065 & ton of $\mathrm{CO}_{2}$-eq \\
\hline \multicolumn{2}{c}{ Total GHG emission } & -3.659 & ton of $\mathrm{CO}_{2}$-eq
\end{tabular}

Chen and Lin [23] found that, amount of GHG transportation of waste collection for composting purpose were 176.53 tons carbon equivalent (MTCE) in Taipei, Taiwan. In compost plant, amount of GHG emission from the treatment of waste (waste degradation and plant maintenance) were 149.75 MTCE in Taipei, Taiwan [23].

\section{Interpretation}

The result from the life cycle assessment of composting in the CCC shows that composting is highly advantageous solid waste treatment method for reducing greenhouse effects. Composting can also reduce volume of waste in an 
environment friendly way. If the CGTP is expanded with gas $\left(\mathrm{CH}_{4}\right)$ collection system, the expanded system could become more favorable than present composting facility in the global warming point of view. Then, collected gas could be used for domestic purposes instead of natural gas. Through improving further facilities, composting could become a supplementary source for electricity generation by utilizing collected gas. Because, it was proved that, the installment of methane $\left(\mathrm{CH}_{4}\right)$ collection facilities in compost plant is becoming a green source of energy. This installment not only reduces GHG emission, but also recovers methane $\left(\mathrm{CH}_{4}\right)$ which can be successfully utilized for electricity production $[2,8$, $10,18,37,57]$.

\section{Conclusions}

The study reveals that the existing municipal solid waste management (MSWM) system of the Chittagong City Corporation (CCC) is not climate friendly. It is a significant contributor to the global climate change, because it releases huge amount of greenhouse gas (GHG) from different solid waste management activities. The result from the assessment indicates that, composting and recycling are climate friendly among municipal solid waste (MSW) management activities of the CCC. These two methods have a positive contribution in reducing GHG emission. Notwithstanding this, transportation and land-filling of MSW are responsible for GHG emission. However, the study will be useful for strategy development on climate change mitigation in Bangladesh. 


\section{References}

[1] D. Suocheng, K. W. Tong and W. Yuping, Util. Policy, 2001, 10(1), 7.

[2] E. Cifrian, A. Andres and J. Viguri, Waste Biomass Valori., 2013, 4(2), 271.

[3] IPCC, 2006 "IPCC Guidelines for National Greenhouse Gas Inventories", Prepared by the National Greenhouse Gas Inventories Programme, IGES, Kanagawa, Japan, 2006.

[4] S. Manfredi, D. Tonini, T. Christensen and H. Scharff, Waste Manage. Res.,2009, 27(8), 825.

[5] R. Pipatti and M. Wihersaari, Mitig. Adapt. Strat. Gl. Chang., 1998, 2(4), 337.

[6] M. Pöschl, S. Ward and P. Owende, Appl. Energ., 2010, 87(11), 3305.

[7] W.T. Tsai, Renew. Sust. Energ. Rev., 2007, 11(2), 331.

[8] J. Dong, M. Ni, Y. Chi, D. Zou and C. Fu, Environ. Sci. Poll. Res., 2013, 20(8), 5512.

[9] A. Yee, M. Brostrom and C. Felske, "Climate Change and Cities : Mitigation through the effective management of Waste, In: A. Khare and T. Beckman (Eds.)", Springer Berlin Heidelberg, 2013.

[10] O. Ayalon, Y. Avnimelech and M. Shechter, Environ. Manage., 2001, 27(5), 697.

[11] R. Pipatti and I. Savolainen, Energ. Convers. Manage., 1996, 37(6-8), 1105.

[12] C. Liamsanguan and S. Gheewala, J. Environ. Manage., 2008, 87(1), 132.

[13] W. Qin, F. Egolfopoulos and T. Tsotsis, Chem. Eng. J., 2001, 82(1-3), 157. 
[14] J. Bogner, M. A. Ahmed, C. Diaz, A. Faaij, Q. Gao, S. Hashimoto, K. Mareckova, R. Pipatti and T. Zhang, Waste Management, in: B. Metz, O. R. Davidson, P. R. Bosch, R. Dave, and L. A. Meyer (Eds.), Cambridge University Press, Cambridge, UK and New York, USA, 2007.

[15] S. Kumar, S. Gaikwad, A. Shekdar, P. Kshirsagar and R. Singh, Atmos. Environ., 2004, 38(21), 3481.

[16] USEPA (United States Environmental Protection Agency), "Global anthropogenic non- $\mathrm{CO}_{2}$ greenhouse gas emissions: 1990-2030”, 2017.

[17] IPCC, "Climate Change 2001: Mitigation. Contribution of Working Group III to the Third Assessment Report of the Intergovernmental Panel on Climate Change”, Cambridge University Press, Cambridge, UK, 2001.

[18] X. Xin-Hua, Y. Yue-Ping and W. Da-Hui, J. Zhejiang Univ. Sci., 2003, $4(3), 352$.

[19] U. Hegde, T. Chang and S. Yang, Chemosphere, 2003, 52(8), 1275.

[20] C. Chiemchaisri, J. Juanga and C. Visvanathan, Environ. Monit. Assess., 2007, 135(1-3), 13

[21] K. Wangyao, S. Towprayoon, C. Chiemchaisri, S. H. Gheewala and A. Nopharatana, Environ. Monit. Assess., 2010, 164(1-4), 13.

[22] V. Talyan, R. Dahiya, S. Anand and T. Sreekrishnan, Resour. Conserv. Recycl., 2007, 50(3), 240.

[23] T. Chen and C. Lin, J. Hazard. Mater., 2008, 155(1-2), 23.

[24] F. Cherubini, S. Bargigli and S. Ulgiati, Energy, 2009, 34(12), 2116.

[25] J. Hong, X. Li and C. Zhaojie, Waste Manage., 2010, 30(11), 2362. 
44 Md. Danesh Miah and M Abubokor Siddik

[26] T. Matsuda, J. Yano, Y. Hirai and S. Sakai, Int. J. Life Cycle Assess., 2012, 17(6), 743.

[27] A. Mohareb, M. Warith and R. Diaz, Resour. Conserv. Recycl. 2008, 52(11), 1241.

[28] S. Yi, K. H. Kurisu and H. Keisuke, Int. J. Life Cycle Assess., 2011, 16(7), 652.

[29] W. Zhao, E. van der Voet, Y. Zhang and G. Huppes, China. Sci. Total Environ., 2009, 407(5), 1517.

[30] M. Banar, Z. Cokaygil and A. Ozkan, Waste Manage. 2009, 29(1), 54.

[31] S. Consonni, M. Giugliano, A. Massarutto, M. Ragazzi and C. Saccani, Waste Manage., 2011, 31(9-10), 2057.

[32] A. Craighill and J. Powell, Resour. Conserv. Recycl., 1996, 17(2), 75.

[33] S. Menikpura, S. Gheewala, S. Bonnet and C. Chiemchaisri, Waste Biomass Valori., 2013, 4(2), 237.

[34] S. Inazumi, H. Ohtsu, T. Shiotani and T. Katsumi, J. Mater. Cycles Waste Manage., 2011, 13(2), 139.

[35] C. Liamsanguan and S. Gheewala, J. Clean. Prod., 2008, 16(17), 1865.

[36] J. Ménard, P. Lesage, L. Deschênes and R. Samson, Int. J. Life Cycle Assess., 2004, 9(6), 371.

[37] L. Bastian, J. Yano, Y. Hirai and S. Sakai, J. Mater. Cycles Waste Manage., 2013, 15(1), 49.

[38] C. Liamsanguan and S. Gheewala, Int. J. Life Cycle Assess., 2007, 12(7), 529. 
[39] H. Merrild, A. Damgaard and T. Christensen, Resour. Conserv. Recycl., 2008, 52(12), 1391.

[40] BMDF, "Study on municipal solid waste management", 2014.

[41] R. Chowdhury, M. Sujauddin, S. Murakami, P. Chakraborty and M. Alam, Int. J. Environ. Waste Manage., 2013, $12(2), 167$.

[42] I. Enayetullah, A. Sinha and S. Khan, "Urban Solid Waste Management Scenario of Bangladesh: Problems and Prospects". Dhaka Waste Concern Technical Documentation, 2005.

[43] M. Salam, M. Hossain, S. Das, R. Wahab and M. Hossain, Int. J. Environ. Sci. Manage. Eng. Res., 2012, 1(4), 160.

[44] Waste Concern, Waste database of Bangladesh, 2014.

[45] M. Alamgir and A. Ahsan, Iran. J. Environ. Health Sci. Eng., 2007, 4(2), 67.

[46] Q. H. Bari, K. M. Hassan and M. E. Haque, Waste Manage., 2012, 32(11), 2029.

[47] M. Sujauddin, S. Rahman and S. Murakami, "Existing solid waste recycling system of Bangladesh and its future". Thirteenth International Waste Management and Landfill Symposium, S. Margherita di Pula, Cagliari, Italy, CISA Publisher, 2011.

[48] K. M. Bahauddin and M. H. Uddin, J. Environ. Sci. Nat. Resour., 2012, 5(1), 99.

[49] BBS, "Statistical Year Book of Bangladesh-2011”, Ministry of Planning, Government of the People's Republic Bangladesh, Dhaka, 2012. 
[50] BBS, "Population and Housing Census 2011". Dhaka. Ministry of Planning, Government of the People s Republic Bangladesh, 2012.

[51] IGES, GHG Calculator for Solid Waste, Version II-2013, 2014.

[52] A. Shekdar, Waste Manage., 2009, 29(4), 1438.

[53] A. M. Troschinetz and J. R. Mihelcic, Waste Manage., 2009, 29(2), 915.

[54] M. Sharholy, K. Ahmed, G. Mahmood and R. Trivedi, Waste Manage., 2008, 28(2), 459.

[55] C. K. Vidanaarachchi, S. T. Yuen and S. Pilapitiya, Waste Manage., 2006, 26(8), 920.

[56] K. A. Weitz, S. A. Thorneloe, S. R. Nishtala, S. Yarkosky and M. Zannes, J. Air Waste Manage. Assoc., 2002, 52(9), 1000.

[57] N. P. Thanh and Y. Matsui, Environ. Monit. Assess., 2013, 185(10), 7993.

Manuscript received on 25 September, 2016, Revised manuscript received on 16 August, 2017 and accepted on 17 September, 2017

The Chittagong Univ. J. Sc. Vol. 40, 2018 